\title{
Correction to: Smoke-saturated Water and Karrikinolide Modulate Germination, Growth, Photosynthesis and Nutritional Values of Carrot (Daucus carota L.)
}

\author{
Arshiya Akeel ${ }^{1} \cdot$ M. Masroor Akhtar Khan ${ }^{1} \cdot$ Hassan Jaleel $^{1} \cdot$ Moin Uddin $^{2}$
}

Published online: 25 September 2019

(c) Springer Science+Business Media, LLC, part of Springer Nature 2019

\section{Correction to: Journal of Plant Growth Regulation https://doi.org/10.1007/s00344-019-09941-w}

The original version of this article unfortunately contained a mistake in Table 3. The correct Table 3 is given below:

Table 3 Effect of pre-soaking seed treatment with smoke saturated water (SSW) and karrikinolide $\left(\mathrm{KAR}_{1}\right)$ on root length, root diameter, root fresh and dry weight of Daucus carota L. recorded at 120 DAS (days after sowing)

\begin{tabular}{|c|c|c|c|c|}
\hline \multirow[t]{2}{*}{ Treatments } & \multicolumn{4}{|l|}{ Parameters } \\
\hline & Root length $(\mathrm{cm})$ & Root diameter $(\mathrm{cm})$ & $\begin{array}{l}\text { Root fresh weight } \\
\left(\mathrm{g} \mathrm{plant}^{-1}\right)\end{array}$ & $\begin{array}{l}\text { Root dry } \\
\text { weight (g } \\
\left.\text { plant }^{-1}\right)\end{array}$ \\
\hline \multicolumn{5}{|c|}{ SSW concentrations } \\
\hline Control (DDW) & $12.10 \pm 0.15^{\mathrm{e}}$ & $2.81 \pm 0.03^{\mathrm{e}}$ & $34.28 \pm 1.21^{\mathrm{d}}$ & $8.57 \pm 0.06^{c}$ \\
\hline $25.8 \mu \mathrm{g} \mathrm{L}^{-1}$ & $14.40 \pm 0.15^{\mathrm{b}}$ & $3.53 \pm 0.02^{\mathrm{ab}}$ & $44.63 \pm 1.11^{\mathrm{ab}}$ & $12.05 \pm 0.06^{\mathrm{b}}$ \\
\hline $51.6 \mu \mathrm{g} \mathrm{L}^{-1}$ & $16.60 \pm 0.12^{\mathrm{a}}$ & $3.75 \pm 0.01^{\mathrm{a}}$ & $48.59 \pm 1.92^{\mathrm{a}}$ & $12.26 \pm 0.02^{\mathrm{a}}$ \\
\hline $103.2 \mu \mathrm{g} \mathrm{L}^{-1}$ & $13.50 \pm 0.10^{\mathrm{c}}$ & $3.35 \pm 0.01^{\mathrm{c}}$ & $40.78 \pm 1.59 b^{c}$ & $9.78 \pm 0.05^{\mathrm{c}}$ \\
\hline $258.0 \mu \mathrm{g} \mathrm{L}^{-1}$ & $12.80 \pm 0.21^{\mathrm{d}}$ & $3.23 \pm 0.02^{\mathrm{d}}$ & $37.24 \pm 1.19 \mathrm{c}^{\mathrm{d}}$ & $9.66 \pm 0.03^{c}$ \\
\hline \multicolumn{5}{|c|}{$\mathrm{KAR}_{1}$ concentrations } \\
\hline Control (DDW) & $12.10 \pm 0.15^{\mathrm{e}}$ & $2.81 \pm 0.03^{\mathrm{e}}$ & $34.28 \pm 1.21^{\mathrm{d}}$ & $8.57 \pm 0.06^{\circ}$ \\
\hline $0.015 \mu \mathrm{g} \mathrm{L}^{-1}$ & $15.70 \pm 0.12^{\mathrm{c}}$ & $3.65 \pm 0.02^{\mathrm{c}}$ & $42.60 \pm 1.14^{\mathrm{bc}}$ & $10.65 \pm 0.04^{c}$ \\
\hline $0.150 \mu \mathrm{g} \mathrm{L}^{-1}$ & $16.60 \pm 0.10^{\mathrm{b}}$ & $3.72 \pm 0.01^{\mathrm{b}}$ & $45.78 \pm 1.26^{\mathrm{b}}$ & $10.99 \pm 0.05^{\mathrm{b}}$ \\
\hline $1.501 \mu \mathrm{g} \mathrm{L}^{-1}$ & $17.20 \pm 0.15^{\mathrm{a}}$ & $3.97 \pm 0.03^{\mathrm{a}}$ & $52.65 \pm 1.04^{\mathrm{a}}$ & $14.21 \pm 0.03^{\mathrm{a}}$ \\
\hline $15.013 \mu \mathrm{g} \mathrm{L}^{-1}$ & $13.20 \pm 0.26^{\mathrm{d}}$ & $3.42 \pm 0.02^{\mathrm{d}}$ & $39.34 \pm 1.10^{\mathrm{c}}$ & $10.23 \pm 0.02$ \\
\hline
\end{tabular}

Each value represents the mean of five replicates written with \pm S.E. Means within a row followed by the same letter(s) are not significantly different $(p<0.05)$

$D D W$ double distilled water
The original article can be found online at https://doi.org/10.1007/ s00344-019-09941-w.

Arshiya Akeel

arsh.aqeel@gmail.com

1 Department of Botany, Aligarh Muslim University, Aligarh, India

2 Botany Section, Women's College, Aligarh Muslim University, Aligarh, India
Publisher's Note Springer Nature remains neutral with regard to jurisdictional claims in published maps and institutional affiliations. 